\title{
Management of Migraine and the Accessibility of Specialist Care - Findings from a Multi-national Assessment of 28 Healthcare Networks
}

Pinay Kainth, ${ }^{1}$ Niamh Murphy, ${ }^{2}$ Silvia Rossi, ${ }^{3}$ Jean-Noël Talabardon, ${ }^{4}$ Marco Pedrazzoli, ${ }^{5}$ Leonardo Mascagni ${ }^{5}$ and Irene Menarini ${ }^{5}$

1. Novartis Pharmaceuticals Canada Inc., Dorval, Quebec, Canada; 2. Novartis Ireland Limited, Dublin, Ireland; 3. Novartis Farma S.p.A, Origgio (VA), Italy;

4. Novartis Pharma AG, Basel, Switzerland; 5. LSC Lifesciences Consultants, Milan, Italy

ntroduction: Migraine is one of the leading reasons for patient access to neurology services. Waiting lists can limit patients' ability to access specialist care, even at specialised headache centres. Our study aims to investigate this issue, identify possible root causes and also document existing good practices. Methods: We conducted a study in a sample of 28 headache centres and their networks in six countries by performing in-depth interviews with 166 healthcare professionals. Results: The waiting list for new patients and follow-up visits exceeded 3 months in 61\% and 36\% of centres, respectively. Patients waited on average 6 months for their first consultation, with peaks beyond 12 months. Five areas were identified as common root causes: (1) inappropriate referral of patients with low-frequency episodic migraine or patients under acute treatment, (2) lack of triage/priority allocation, (3) limited resource availability or resources dedicated to migraine, (4) limited delegation of activities, and (5) suboptimal management of follow-up visits. Conclusion: Our work highlights a gap between best practices for migraine management proposed in the literature and current real-world practice. Guidelines recommend a "network" approach to bridge different levels of care. Based on our findings, consistency in practice amongst specialised headache clinics and integration with primary care represent an important area for further improvement.

\section{Keywords}

Migraine, waiting lists, access, care, network, practice

Disclosures: Pinay Kainth is employed by Novartis Pharmaceuticals Canada Inc in Canada. Niamh Murphy is employed by Novartis Ireland Limited in Ireland. Silvia Rossi is employed by Novartis Farma S.p.A in Italy. Jean-Noël Talabardon is employed by Novartis Pharma AG in Switzerland. Marco Pedrazzoli, Leonardo Mascagni and Irene Menarini are employed by Lifesciences Consultants in Italy. Review Process: Double-blind peer review.

Acknowledgements: We are thankful to all the healthcare professionals who participated in the study for their availability and insight. The following centres/private practices participated in this study: Austin Hospital Melbourne (Melbourne, Australia), Migraine Specialist (Brisbane, Australia), Sunshine Coast University Hospital (Birtinya, Australia), The Department of Clinical Neurosciences - University of Calgary (Calgary, Canada), The Shapero Markham Headache and Pain Treatment Centre Markham (Markham, Canada), Ottawa Headache Centre Research (Ottawa, Canada), Charité Hospital (Berlin, Germany), Private Practice (Hamburg, Germany), Private Practice (Essen, Germany), Private Practice (Koln, Germany), DRK Kassel Krankenhaus (Kassel, Germany), Beckerklinik (Krozingen, Germany), St. Vincent's University Hospital (Dublin, Ireland), Bon Secours Hospital (Cork, Ireland), Limerick University Hospital (Limerick, Ireland), AO Perugia (Perugia, Italy), AO Sant'Antonio Abate (Varese, Italy), Centro Neurolesi Bonino Pulejo - IRCCS Messina (Messina, Italy), Fondazione Mondino (Pavia, Italy), Ospedale San Vito al Tagliamento (Pordenone, Italy), Ospedale San Martino (Genova, Italy), IRCCS San Raffaele la Pisana (Rome, Italy), AO Seconda Università degli Studi di Napoli (SUN, Italy), Isala Medisch Centrum (Zwolle, Netherlands), Meander Medisch Centrum (Amersfoort, Netherlands), Zoorgsaam Hospital (Terneuzen, Netherlands), Zuyderland Hospital (Sittard, Netherlands). Editorial support was provided by Leonardo Mascagni (Lifesciences Consultants in Zurich), and funded by Novartis Pharma AG.

Compliance with Ethics: This study involves an assessment

of interview questionnaires and did not involve any studies with

human or animal subjects performed by any of the authors.

Authorship: All named authors meet the International Committee

of Medical Journal Editors (ICMJE) criteria for authorship of this

manuscript, take responsibility for the integrity of the work as a whole,

and have given final approval for the version to be published.

Open Access: This article is published under the Creative Commons

Attribution Non-commercial License, which permits any non-commercial

use, distribution, adaptation and reproduction provided the original authors

and source are given appropriate credit. The Authors (c) 2018.

Received: 31 May 2018

Accepted: 13 July 2018

Citation: European Neurological Review. 2018;13(2):103-10

Corresponding Author: Jean-Noël Talabardon, Novartis Pharma AG,

Fabrikstrasse 12, 4002 Basel, Switzerland. E: jean-noel.talabardon@novartis.com
Headache disorders represent a large share of consultations in neurology services. A study conducted by the World Health Organization (WHO) revealed that one-third of all neurological consultations are currently reserved for headache. ${ }^{1}$ Neurology clinics often have long waiting lists, extending to over 1 year for a first consultation. Such waiting times substantially delay patients' access to care. ${ }^{2}$ This phenomenon can partially be explained by a gap between availability of neurologists and demand for their services. For instance, in Ireland, a ratio of one neurologist per 140,625 people has been reported, ${ }^{3}$ where the recommended ratio is 1:70,000. ${ }^{4} \mathrm{~A}$ misalignment between neurologist supply and demand has also been reported in the US. ${ }^{5}$ Excessive referral of patients to specialised tertiary centres is also a common cause of inflated waiting lists. European guidelines recommend that patients with migraine are managed by a network of healthcare professionals (HCPS) composed of three tiers: primary care physicians (PCPS), general neurology clinics and headache clinics. Ninety percent of patients with migraine should be treated within the first tier (PCPS), 9\% should be referred to the second tier, and only $1 \%$ should be referred to specialised headache centres. ${ }^{6}$

Our study further explores the topic of patient access to specialty care in migraine and documents wait times as well as other obstacles to migraine care in the context of real-world practice. All assessments were conducted in 2017

\section{Objectives of the study}

The goal of this study was to conduct an in-depth investigation of roadblocks to accessing specialised migraine care, and to document a comprehensive view of the phenomenon. Three objectives are particularly relevant:

- to investigate obstacles to accessing specialised migraine care and assessing the size of the phenomenon in terms of waiting lists;

- to identify the causes of long waiting lists;

- and to recognise and record good practices in migraine management across a variety of settings and countries. 
Table 1: Sample characteristics by country

\begin{tabular}{|c|c|c|c|c|c|}
\hline \multirow[t]{2}{*}{ Country } & \multicolumn{2}{|l|}{ Setting } & \multicolumn{3}{|c|}{ Size (\# of migraine patients managed) } \\
\hline & Hospital & Office & Small (200-600) & Medium (600-1000) & Large $(>1000)$ \\
\hline Australia & 2 & 1 & 2 & 1 & 0 \\
\hline Canada & 2 & 1 & 0 & 1 & 2 \\
\hline Germany & 3 & 3 & 1 & 2 & 3 \\
\hline Ireland & 4 & 0 & 1 & 2 & 1 \\
\hline Italy & 8 & 0 & 2 & 2 & 4 \\
\hline Netherlands & 4 & 0 & 2 & 2 & 0 \\
\hline TOTAL & 23 & 5 & 8 & 10 & 10 \\
\hline
\end{tabular}

\section{Methods}

\section{Sample}

HCPS from six countries and 28 headache centres (and their networks of referring physicians) participated in the study: three networks inAustralia, three in Canada, eight in Italy, six in Germany, four in Ireland and four in the Netherlands. Countries were chosen based on the interest shown towards the project according to the timelines and respective country's local approval. We included a heterogeneous sample of headache centres and networks, which varied in terms of location, size, presence of dedicated headache days versus general neurology clinics and setting type (neurology departments in general hospitals, specialised headache/migraine units and office-based neurology practices and pain clinics)

A total of 166 HCPS were interviewed in the 2017 study. For each network, we divided the participant types into those who work within a headache centre and those in the periphery who may refer. Interviews at headache centres included the head of the neurology department/headache centre and other department/headache centre neurologists/pain specialists, as well as nurses and administrative staff. To provide an overview of the patient journey to the clinic, emergency room physicians, PCPs and office-based neurologists were also included.

\section{Data gathering methodology}

Data were collected via field interviews. A mix of face-to-face and phone interviews were conducted using questionnaires tailored to each HCP's role. Each in-depth interview lasted between 30-60 minutes. Five common types of biases in data gathering were proactively addressed and strategies to mitigate them were adopted.

\section{- Participant's self-selection bias. Our assessment did not allow} networks to self-select themselves into the study; rather, their interest was evaluated following a specific selection process in each country.

- Single responder bias. Interviewing multiple HCPs within the same network allowed us to evaluate inconsistencies in the information collected and probe them during on-field assessments.

- Single interviewer bias. A pool of nine different interviewers was involved, and field assessments were always conducted by at least two interviewers simultaneously.

- Country interviewer bias. Assessments were performed by different interviewers often from different countries than that of interviewees.

- Sponsor bias. We did not directly or indirectly ask physicians to discuss drugs marketed by the sponsoring pharmaceutical company.

\section{Issue identification}

Before starting the assessments, secondary research in each country was conducted in order to get an initial understanding of country-specific issues. Information collected during the field assessments were benchmarked to findings from secondary research. Recurring themes that were at odds with guidelines were considered relevant issues with respect to barriers for accessing migraine care. At the end of the assessment, a more complete understanding of migraine management allowed for cross-country comparison, which highlighted common traits among networks in specific countries and/or settings.

\section{Results}

\section{The framework of analysis}

After completing the field assessment, we grouped the identified issues along five steps of the patient journey (Figure 1): appropriate patient referral, triage and priority allocation, dedicated clinics and resources, nurse role and task delegation, and follow-up management.

The first area identified was 'appropriate patient referral'. This includes current referral practices amongst PCPS that generate an excessive inflow of unsuitable patients to headache centres. The second issue was 'triage and priority allocation', which relates to allocating different levels of priority to patients based on their disease severity and thus regulating access to the centre and type of services offered. 'Dedicated clinics and resources' denotes the number of HCPs dedicated (or partially dedicated) to migraine care, their time allocation to migraine versus other sub-specialties and the availability of dedicated slots for patients with migraine. 'Nurse role and task delegation' looks at the quantity and type of activities in the headache centre that are delegated to nurses or administrative personnel. 'Follow-up management' refers to decisions on who is responsible for ongoing care of patients with migraine after a confirmed diagnosis (i.e., primary care, general neurology, specialised headache clinics).

HCP interviews, network comparisons and benchmarking against literature and international guidelines allowed us to identify roadblocks and good practices for each of these five areas. Several quantitative parameters were also collected and compared across centres:

- length of waiting list (for both first visit and follow-up);

- total number of patients managed by the centre per year and their breakdown by severity and treatment type;

- distribution of patients by origin of referral;

- number of resources per centre and time dedicated to migraine, calculated as total number of clinics dedicated to the disease;

- and duration of specific sub-tasks within a visit (e.g., demographic data and past history collection, diagnostic visit performance, treatment prescription, patient follow-up). 


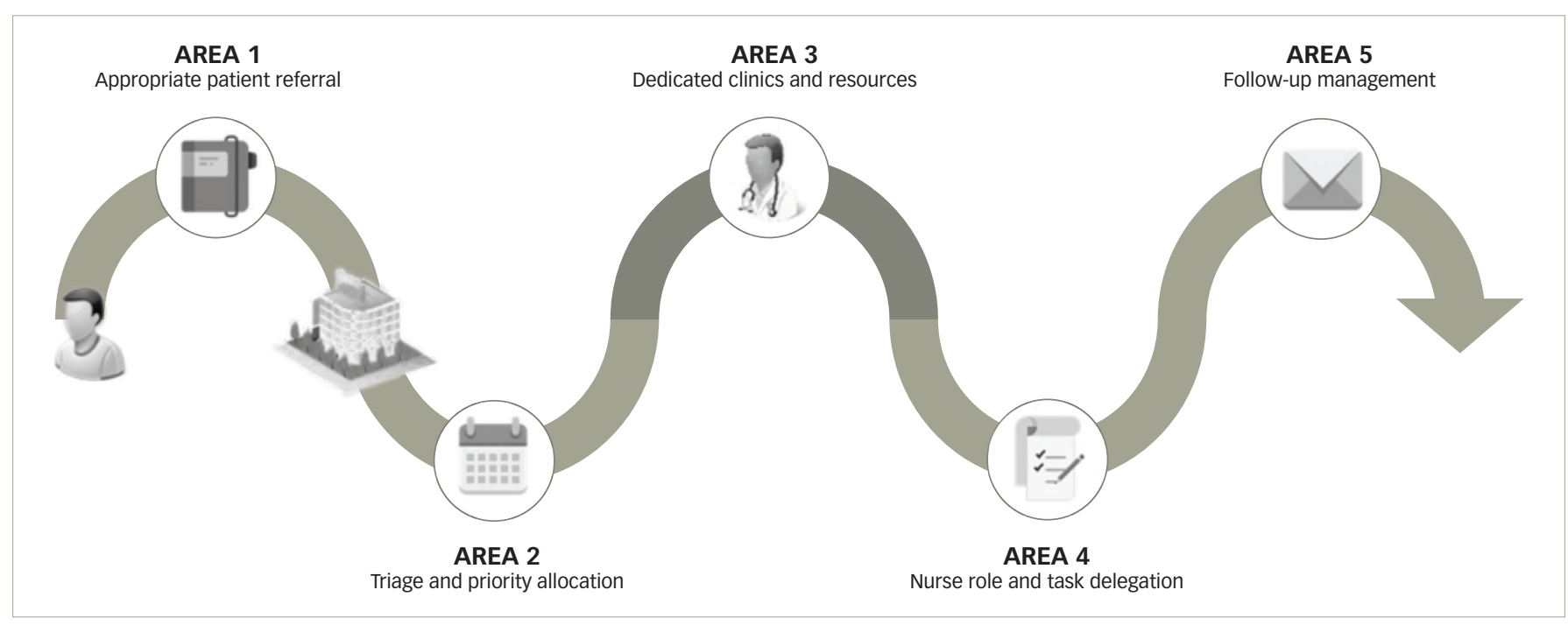

Figure 2: Proportion of centres by waiting list length (in months) for first and follow-up visit
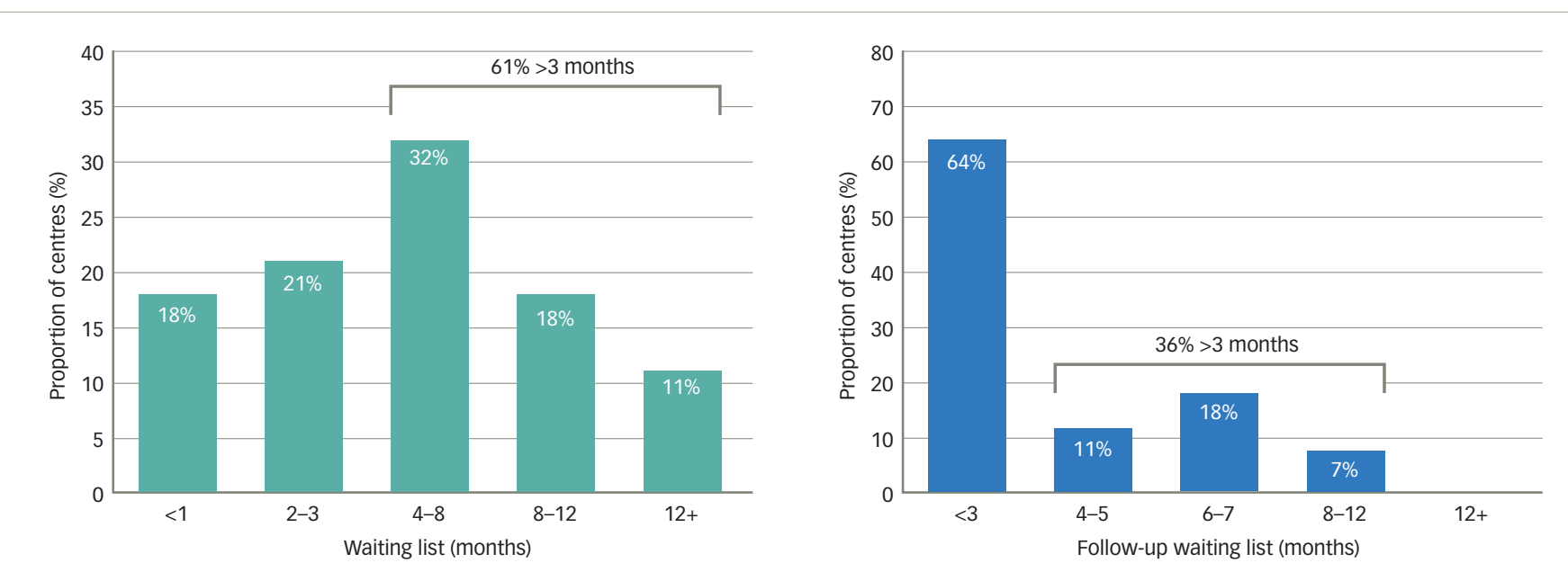

The magnitude of the issue - the size of waiting lists The length of the waiting list to visit a headache centre was used as a measure of patient access to care. $61 \%$ of centres had a waiting list longer than 3 months for a new patient (waiting lists reported for standard visits - urgencies/emergencies were excluded, as they access centres via dedicated fast-tracks). Only $18 \%$ of centres had a waiting list shorter than 1 month for a new patient consultation (range: 1-18 months), while $11 \%$ of headache centres reported more than 1 year of waiting time for a first visit. Waiting lists for follow-ups were on average shorter (range: 0-9 months), although 36\% of centres reported more than a 3-month waiting time for a follow-up visit (Figure 2).

\section{The causes of long waiting lists Appropriate patient referral}

The first cause of long waiting lists that we identified concerned the regulation of patient inflow, i.e., the process and practices for referring patients to a specialised headache centre.

\section{Demographics of patients referred to specialist centres}

Chronic migraine (CM) is a headache occurring on 15 or more days of the month, for more than 3 months, which on at least 8 days per month has the features of migraine headache. ${ }^{7}$ Migraine types not satisfying the above-mentioned criteria (e.g., less than 15 headache days per month) are classified as episodic migraine (EM).? On average, $52 \%$ of patients being referred to headache centres suffered from EM, up to $55 \%$ of which was low frequency EM ( $<4$ migraine days per month). Significant variations were reported across networks in terms of types of patients referred to them (Figure 3). Eight centres had less than 30\% of patients with EM, while five centres recorded a share of patients with EM higher than $80 \%$. For three centres, it was not possible to collect data on the distribution of migraine frequency amongst their patient population. Although frequency of migraine attacks was regarded by physicians as the main criteria driving referral, other disease characteristics, such as functional impact and frequency of acute medication use, should also be taken into consideration.

Initiation of migraine treatment before referral to a specialist centre PCPS often reported a lack of confidence in diagnosing and treating the disease and a general reluctance to recognise and manage medication-overuse headache $(\mathrm{MOH})$. Limited confidence reduced their willingness and ability to manage patients with migraine directly, thus increasing the number of referrals to headache centres. PCPS 


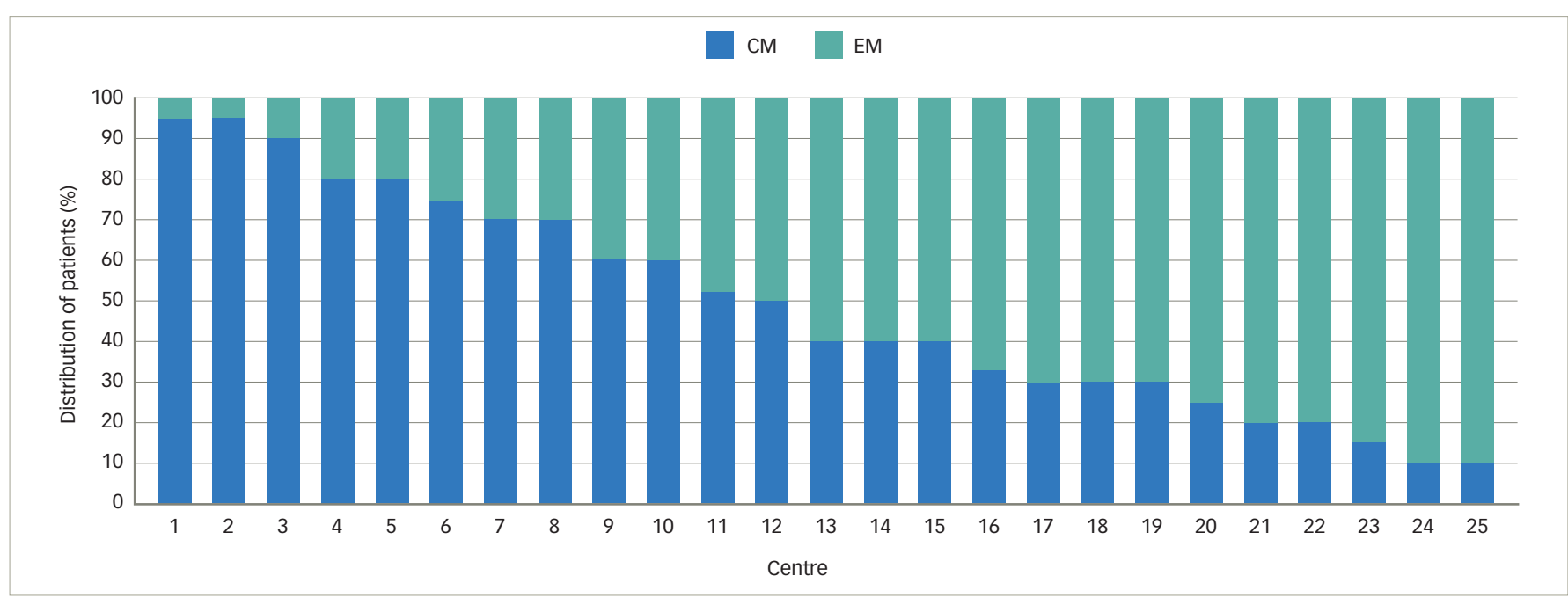

$C M=$ chronic migraine; $E M=$ episodic migraine.

had initiated prophylactic treatment in $66 \%$ of $\mathrm{CM}$ and $22 \%$ of $\mathrm{EM}$ patients who they referred to headache clinics. However, once arriving to headache clinics, $96 \%$ of patients with CM were administered prophylactic treatment after their first visit, while $52 \%$ of patients with EM were prescribed prophylaxis at some point after their first visit.

We identified very different situations across networks in terms of initiation of prophylactic treatment. Networks could be broadly split into two groups: group one (three countries, 17 networks) reported large gaps in the use of prophylaxis before and after patients were referred to a specialised centre. On average, only $53 \%$ of patients referred to the centre with $\mathrm{CM}$ were started on prophylaxis by the referring physician, but a further $46 \%$ ( $99 \%$ of patients in total) were started on prophylaxis after a first visit to the clinic. Only $7 \%$ of referred patients with EM were already on prophylaxis at referral; however, a further $38 \%$ ( $45 \%$ of patients in total) received a prophylaxis prescription in the centre. These data highlight the gap reported by referring physicians regarding their level of confidence with initiating prophylactic treatment on their own.

Networks in group two (three countries, nine networks) displayed a smaller gap in prophylaxis initiation and hence higher efficiency in the referral network. Most patients with CM (88\%) were started on a prophylactic treatment by the referring physician, and a further $5 \%$ were switched to prophylaxis ( $93 \%$ of patients in total) after a first visit in the clinic. Forty-eight percent of referred patients with EM were already on prophylaxis at referral; however, a further $13 \%$ (61\% of patients in total) received a prescription for prophylactic treatment in the centre.

\section{Information sharing and communication}

Ensuring efficient interactions amongst HCPS inside and outside a headache centre can reduce the time patients spend in the clinic. Standard referral letters to facilitate collection of patient history were usually not available and/or used by referring physicians. In $96 \%$ of centres, headache specialists did not have access to patient history before their first visit. According to data collected during interviews, if specialists had access to patient history (e.g., demographics, previous test results and treatments tried) prior to the first visit, consultation time would decrease by 5-10 minutes and allow for an increase in number of total patient visits per day.
A migraine diary is widely recognised as a tool to support neurologists in making an accurate diagnosis. According to the HCPs interviewed, information on headache frequency and severity should be collected by the patient for at least 1 month before a correct diagnosis can be made. Approximately $75 \%$ of patients were referred to clinics without a migraine diary completed before their first visit. In $11 \%$ of cases, a lack of migraine diary resulted in a second visit to decide on an appropriate treatment due to the need to document attack frequency before a treatment plan could be developed.

\section{Triage and priority allocation}

Formal or informal fast-track was available in all networks to ensure that cases indicated as urgent by referring doctors were seen with limited delay. These practices allowed centres to distinguish between urgent cases (e.g., tumour or stroke suspicion, cluster headache) that require accelerated review, and non-urgent cases which were added to the standard waiting list. However, urgent cases accounted for a minor percentage of patients accessing headache centres $(<1 \%)$.

'Advanced triage' activities, distinguishing patients by headache severity, were implemented in $44 \%$ of centres. This triaging allowed neurologists to distinguish patients based on their headache type. Complex cases with high attack frequency (typically patients with high-frequency EM or $\mathrm{CM}$ ) were prioritised over simpler cases with a limited number of attacks per month. In particular, 18\% of centres had a different waiting list based on headache severity. One centre screened patients at referral in order to admit chronic migraineurs only. Eighteen percent of centres relied on triage by severity to allocate more severe cases to specialised migraine clinics and less severe cases to general neurology clinics. Seven percent of centres combined both types of prioritisation. In all other centres, migraineurs were allocated appointments on a 'first-come, first-served' basis, i.e., patients with $\mathrm{CM}$ were not seen on a fast-track basis compared to patients with EM.

\section{Dedicated clinics and resources}

Time dedicated to 'migraine clinic'

We defined migraine clinic as a time slot dedicated to patients with migraine with average duration of 4 hours (either morning or afternoon). Seven centres ( $25 \%$ of total sample) had no clinics dedicated to migraine, resulting in patients being attended to in general neurology clinics. The majority of centres organised 1-3 migraine clinics per week, while 


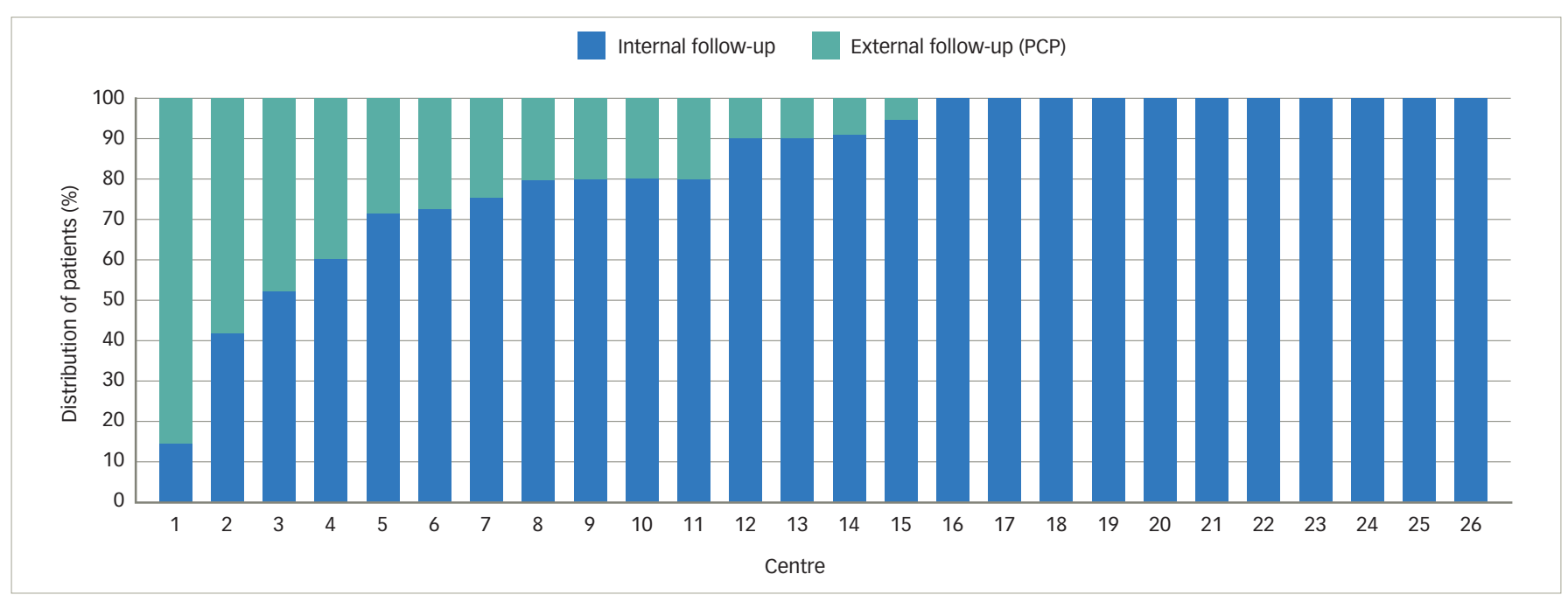

$P C P=$ primary care physician

five centres had 4-8 clinics per week. Only six centres (21\%) had 9-10 migraine clinics per week, that is, a migraine specialist was available on any day from Monday to Friday. Overall, it was observed that in many centres where a migraine clinic existed, HCPs involved in such clinics still dedicated more time to other neurological diseases (e.g., epilepsy and multiple sclerosis) than to migraine.

\section{Existence of dedicated resources}

HCP availability varied widely from centre to centre, ranging between 1-8 neurologists and 0-8 nurses caring for patients with headache per centre. In a third of centres, only one neurologist is responsible for patients with headache. We computed the ratio of monthly neurologists' time dedicated to migraine over total neurologists' clinic time, thus calculating the number of full-time equivalent (FTE) neurologists per centre. Twenty percent of centres had less than 0.2 FTE neurologists, while 65\% reported 0.2-0.4 FTE neurologists. Fifteen percent of centres had 0.5-1 FTE neurologist (only two centres had one FTE neurologist, meaning one neurologist is available in the centre every day of the week Monday to Friday).

\section{Nurse role and task delegation}

\section{Allocation of tasks among different healthcare professionals}

Fifteen percent of centres did not have a dedicated headache nurse. considering centres with headache nurses available, in $38 \%$ of centres, nurses are delegated only basic clinical and administrative activities while in $33 \%$ of centres, they also take care of triaging and patient education. In $29 \%$ of centres, nurses are involved in advanced clinical activities, such as initial patient assessment, diagnosis and follow-up visits. Limited task delegation was reported in some countries because of legislative limitations. However, different levels of nurse engagement in patient management were observed within the same country, suggesting the opportunity for every network to re-evaluate the role of nurses in caring for patients with migraine. ${ }^{8}$

\section{Duration of visits}

Average duration for first consultation was 42 minutes (range: 15-75 minutes). Variability was also observed in the duration of follow-up, with values ranging from 10-40 minutes and a mean length of 21 minutes. Such heterogeneity in visit duration is consistent with previous studies of headache centres' efficiency in Italy ${ }^{9}$ where patients did not perceive visit length as a factor impacting quality of care.

\section{Proactive agenda management}

None of the study centres differentiated visit time by disease severity. Actual visit duration, however, varied across patient types resulting in 'lost' clinical time in cases where allotted visit time was in excess. In more than half of our sample, no patient appointment reminder was in place and issues of no-shows were not addressed. No-show rates were highly variable, with a recorded minimum of $0 \%$ up to a maximum of $35 \%$ of patients not showing up for appointments. The average was $6 \%$.

\section{Follow-up management}

\section{Modality and setting of follow-up visits}

Eighty-four percent of patients referred to headache centres stayed with the clinic for at least one follow-up visit. Large variations in follow-up practices were observed, with a minimum of $15 \%$ of patients followed up in the clinic once referred and a maximum of 100\% (Figure 4). For two centres it was not possible to collect data on the distribution by follow-up setting.

\section{Frequency of follow-up visits}

On average, patients underwent three follow-up visits per year with the specialist centre. Eighty-eight percent of centres performed two to four follow-up visits per year, while different frequencies were observed for the remaining $12 \%$ of centres. Of these, one centre reported doing six follow-up visits per year (every 2 months), while two centres organised follow-up visits every 9 months (patients underwent a mean of one follow-up visit per year). It is important to note that these numbers are average values. Some patients, for example those experiencing side effects, were visited with a higher frequency.

\section{Coordination of different levels of care}

In our study, four different network archetypes were identified (Figure 5). In 'centralised networks' (27\% of total networks), a centre of reference attracts referrals from PCPS or general neurologists outside the catchment area and patients are then followed up by the specialised unit. In 'decentralised networks' (23\% of total networks), a centre of reference attracts referrals from PCPS or neurologists in the area and simpler cases are usually discharged back to referring physicians. In 'incomplete networks' (20\%), a headache centre is missing and patients are usually referred to general neurologists. 'Multilevel networks' (30\%) are composed of a recognised tertiary care unit managing only complicated cases sent by PCPS or general neurologists. 

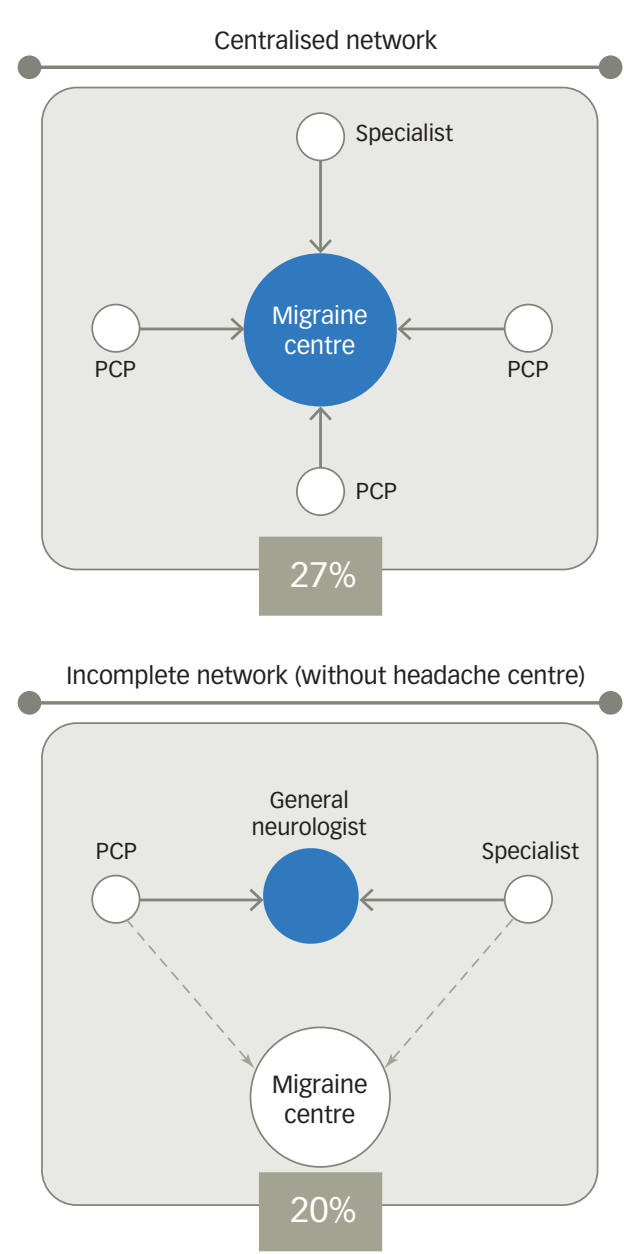
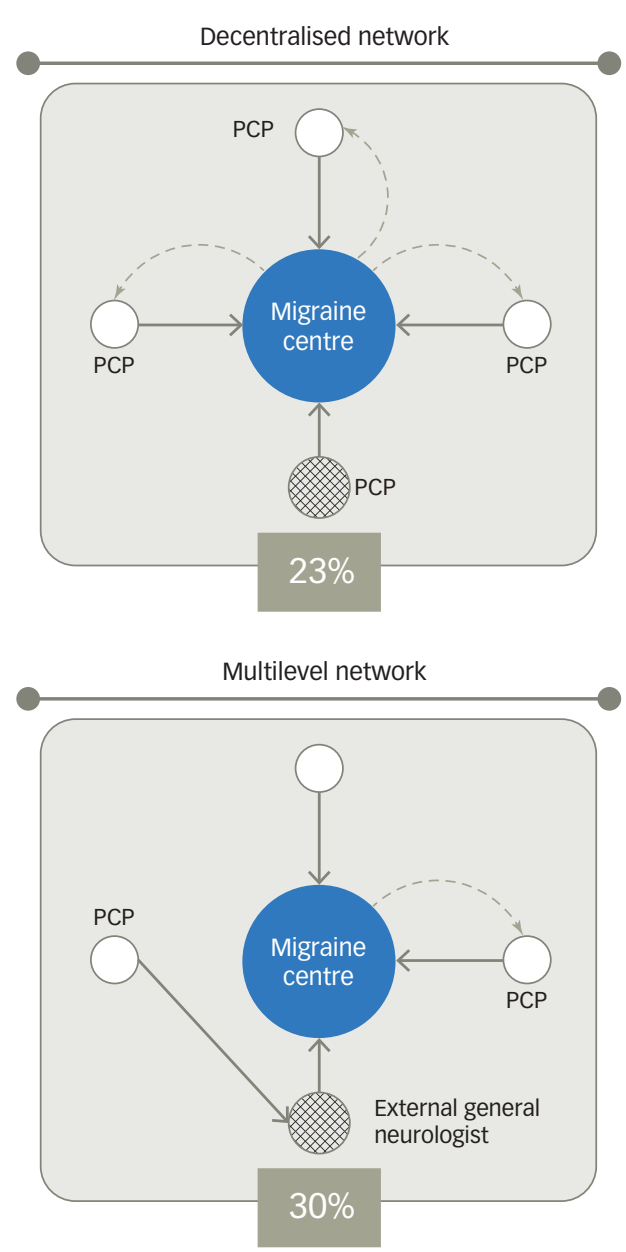

$P C P=$ primary care physician

\section{Discussion and conclusions}

Guidelines suggest that access to migraine care in specialised centres should be granted to all patients with $\mathrm{CM}^{10,11}$ Previous studies suggest that $<5 \%$ of patients with $\mathrm{CM}$ are able to go through the three barriers to receiving care for headache (consultation, diagnosis and treatment). ${ }^{12}$ This is mainly due to long waiting lists as the barrier to accessing specialised care. ${ }^{13}$ Our study confirmed that waiting lists in numerous headache centres are an issue. Patients wait on average 6 months for a first/new patient visit (in some cases up to 18 months). Waiting lists were longer than 3 months in $61 \%$ and $36 \%$ of centres for first visits and follow-up visits respectively, delaying or even limiting patients' access to care. Patients and/or referring physicians were reported to avoid referral to specialised centres with waiting lists over 1 year, negatively impacting patients' ability to receive appropriate specialist care.

A three-tier approach to patient management is recommended to improve efficiency of care. ${ }^{6}$ PCPS should manage the majority of patients, managing acute treatment and starting first-line prophylaxis. ${ }^{14}$ In our study, only $66 \%$ and $22 \%$ of patients with $\mathrm{CM}$ and EM, respectively, were referred to the specialised centre having already received at least one prophylactic treatment. Hence an excessive referral of patients from primary care directly to specialised tertiary headache centres was observed. Furthermore, patients with EM constituted half of referrals to specialised centres, a high share of them (up to 55\%) being patients with low-frequency migraine, who remain on acute treatment only, even after a visit to the headache clinic. We also detected reluctance to tackle $\mathrm{MOH}$ in primary care settings: $34 \%$ of patients referred to specialised units had $\mathrm{MOH}$ and did not receive a prior attempt to manage $\mathrm{MOH}$ by referring physicians. This finding is important because simply giving patients information and advice about $\mathrm{MOH}$ has been shown to reduce headache frequency and acute medication use in many patients with $\mathrm{MOH} .{ }^{15}$

Evidence from centres in our study suggests good practices can be implemented to improve the appropriateness of referrals. Some headache centres have started to train referring physicians and provide them with documentation to support referral. Similarly, a study by Braschinsky et al. has shown how a structured but simple training programme could lead to better headache practice amongst PCPS. ${ }^{16}$

The importance of triage to prioritise patients and efficiently manage the demand for consultation has been emphasised in the literature. ${ }^{17}$ According to our findings, basic formal or informal triage was performed in all centres to ensure patients with acute attacks or with tumour/stroke suspicion could skip standard waiting lists and be seen urgently. However, only 12 centres implemented more advanced triage procedures aimed at differentiating access priorities among non-urgent cases, who represent the vast majority of the case load. Such procedures help guarantee access to severe cases and in these 12 centres they helped limit waiting lists for patients with $\mathrm{CM}$ to 3 months, on average. 
A lack of dedicated clinics and resources for migraine was reported during our study as one of the leading causes of long waiting lists. These resources were reportedly lower than what was allocated in many centres to other sub-specialties such as epilepsy or multiple sclerosis, despite higher prevalence of migraine. Evidence from centres dedicating resources specifically to migraine (dedicated time slots) suggests the set-up of migraine-dedicated clinics is an important first step to ensure patient access to specialised care.

An OECD (Organisation for Economic Cooperation and Development) study ${ }^{18}$ also showed that more than $75 \%$ of doctors and nurses from multiple divisions perceived themselves to be over-skilled for some of their assigned job tasks. Comparison across headache centres showed significant differences in the role of nurses, ranging from very limited engagement in the care of patients with migraine to significant levels of independence in performing tasks such as the initial part of first visits as well as full follow-up visits. Some of the centres assessed showed how delegating activities to nurses can impact the overall efficiency of clinics and the size of patient pool manageable given the set of resources. In two centres, nurses supported patients over the phone with medication adjustments (in cases of no therapeutic response, they could change doses and drugs). A study by Blumenfeld et al. highlighted how a comprehensive treatment plan for patients with migraine, developed by a nurse practitioner in coordination with the patient, resulted in improved patient satisfaction and a reduction in overall healthcare utilisation. ${ }^{19}$ Delegation of activities to nurses increases specialists' capacity to see patients and thus, could reduce waiting lists.

Follow-up of patients should be managed using a three-tiered approach to reduce the burden on specialised headache centres. ${ }^{6,20} \mathrm{An}$ integrated healthcare approach can result in more efficient and, in some cases, more cost-effective management of patients with migraine. ${ }^{21}$ According to our study however, follow-up visits were performed in the headache centre for $84 \%$ of patients on average. A lack of confidence with migraine management amongst PCPs limits the share of patients referred back to them after a visit to a headache clinic. We did observe a positive impact in clinics where efficient follow-up management was practiced. In centres with three levels of care, waiting lists were on average 3 months for a first visit and 1 month for follow-ups.

Finally, our study highlights that, while a few internal practices do increase the ability of a headache centre to provide timely access to care for patients, most improvements require cooperation between HCPS in the centres and in the network (PCPS, other neurologists). The variation in waiting lists, resources and activities among the networks involved in our study demonstrates that HCPs have a chance to tailor their clinical practice and promote faster access to specialty care for patients.

\section{Limitations and implications for further studies}

We acknowledge the existence of limitations to this study, which suggest further exploration on this topic. Sample size is relatively limited given the variations observed in migraine management practices. Twenty-eight networks in six different countries allowed us to include very different types of practices (e.g., private neurology clinics, public general hospitals, office-based practices), but may not represent each of these variations at a country level. The inclusion of practices and countries in the sample was not based on statistical considerations: practices might not be representative of a country situation and results in the selected countries might not be directly applicable to other countries.

Biases in data collection are implicit in an interview-based study, despite the strategies we implemented to mitigate them. Combining interviews with analyses of databases internal to centres could provide more robust data and additional insights. A study of the correlation between the key variables identified in this study and waiting lists for patients with migraine should be performed, with a sample large enough to statistically confirm the evidence from our study, while controlling for confounding factors.

Patient perception of disease management and treatment were not taken into account for the purposes of the study. Specific analyses on satisfaction with migraine care could provide further information on the topic.

The network-based approach should be further expanded to probe the existence of other network archetypes and different referral dynamics. This could disclose additional insights about patient management and highlight other possible issues and good practices.

A more in-depth study of good practices is also advisable. This type of analysis, for instance, in the form of prospective interventional studies, would be beneficial to understand what options are best for migraine care and patient access to headache centres.
1. World Health Organization. Headache disorders: Fact sheet. 2016. Available at: www.who.int/en/news-room/fact-sheets/ detail/headache-disorders (accessed 23 February 2018).

2. Condon D. Big jump in neurology waiting list. 2014. Available at: www.irishhealth.com/article.html?id=24245 (accessed 23 February 2018)

3. The Dublin Neurological Institute. Available at: www. neurologicalinstitute.ie/about-the-neurological-institute/proftim-lynch-consultant-neurologist (accessed 23 February 2018).

4. Neurological Alliance of Ireland. Report on national survey of neurology services. 2015. Available at: www.nai.ie/assets/22/ E622B527-41E1-4471-9EFDAE484E1745B2_document/ NAI_Report_on_National_Services_2015_C.pdf (accessed 24 July 2018)

5. Dall T, Storm MV, Chakrabarti R, et al. Supply and demand analysis of the current and future US neurology workforce. Neurology. 2013:81:470-8.

6. Steiner TJ, Antonaci F, Jensen R, et al. Recommendations for headache service organisation and delivery in Europe. Jeadache Pain. 2011;12:419-26.

7. Headache Classification Committee of the International Headache Society (IHS). The International Classification of Headache Disorders, 3rd edition. Cephalalgia. 2018;38:1-211.

8. Leroux E, Beaudet L, Boudreau $\mathrm{G}$, et al. A nursing intervention increases quality of life and self-efficacy in migraine: a 1-yea prospective controlled trial. Headache 2018:58:260-74. Pellesi L, Benemei S, Favoni V, et al. Quality indicators in headache care: an implementation study in six Italian specialist-care centres. J Headache Pain. 2017:18:55.

10. British Association for the Study of Headache (BASH) Guidelines for all healthcare professionals in the diagnosis and management of migraine, tension-type headache, cluste headache and medication-overuse headache. 3rd edition (1st revision). 2010. Available at: www.bash.org.uk/wp-content/ uploads/2012/07/10102-BASH-Guidelines-update-2_v5-1-indd. pdf (accessed 24 July 2018).

11. Società Italiana per lo Studio delle Cefalee (SISC). Linee guida per la diagnosi e la terapia delle cefalee primarie. 2011. Available at wmw.siscit/ita/linee-guida-diagnostico2011. Avallable at: Www.sisc.it/ta//inee-guida-diagnosticoterapeutiche-delle-cefalee-primarie_33.html (accessed 24
July 2018).

2. Moriarty M, Mallick-Searle T. Diagnosis and treatment for chronic migraine. Nurse Pract. 2016;41:18-32.

13. Dodick DW, Loder EW, Manack Adams A, et al. Assessing barriers to chronic migraine consultation, diagnosis, and treatment: results from the Chronic Migraine Epidemiology and Outcomes (CaMEO) Study. Headache. 2016;56:821-34.

14. Becker WJ. The diagnosis and management of chronic migraine in primary care. Headache. 2017;57:1471-81.

15. Kristoffersen ES, Straand J, Vetvik KG, et al. Brief intervention for medication-overuse headache in primary care. The $\mathrm{BIMOH}$ study: a double-blind pragmatic cluster randomised parallel controlled trial. J Neurol Neurosurg Psychiatry. 2015;86:505-12.

16. Braschinsky M, Haldre S, Kals M, et al. Structured education can improve primary-care management of headache: the first empirical evidence from a controlled interventional study. J Headache Pain. 2016;17:24.

17. Nijjar S, Pink L, Gordon AS, Examination of migraine management in emergency departments. Pain Res Manag. 2011;16:183-6

18. Colombo F. Health workforce policies: right jobs, right skills, in the right places: The 6th International Jerusalem Conference on Health Policy. 2016. Available at: www.oecd.org/health/ health-systems/Health-workforce-policies-Colombo-May2016. pdf (accessed 20 July 2018).

19. Blumenfeld A, Tischio M. Centre of excellence for headache care: group model at Kaiser Permanente. Headache. 2003:43:431-40.

20. Gaul C, Brömstrup J, Fritsche G, et al. Evaluating integrated headache care: a one-year follow-up observational study in patients treated at the Essen Headache Centre. BMC Neurology. 2011;11:124.

21. Porter ME, Guth C, Dannemiller EM. The West German Headache Center: integrated migraine care. Harvard Business School Case. 2007;707-559. 


\section{Original Research Headache}

\section{Other literature:}

ers: differentiating and managing the common subtypes. Br J Pain. 2012;6;124-32.

Blumen H, Lenderman T. How using care management guidelines can help accountable care organizations achieve success. 2011. Available at: www.milliman.com/uploadedFiles/insight/healthreform/howusing-care-management.pdf (accessed 24 July 2018).

- Pringsheim T, Davenport W, Mackie G, et al. Canadian headache society guideline for migraine prophylaxis. Can J Neurol Sci. 2012;39(2 Suppl. 2):S1-59.

Holland S, Silberstein S, Freitag F. et al. Evidence-based guideline update: NSAIDS and other complementary treatments for episodic migraine prevention in adults. Neurology. 2012;78:1346-53.

Welsh National Health Service. North Wales Critical Care Network - Annual report 2009-2010. Available at: www.wales.nhs.uk/sites3/Documents/753/Annual\%20Report\%2009-10\%20\%5BFinal\%5D.pdf (accessed 23 February 2018).

- Lipton R, Bigal M, Diamond M, et al. Migraine prevalence, disease burden, and the need for preventive therapy. Neurology. 2007;68:343-9. 